\title{
Capacitación socioafectiva de alumnos y profesores \\ a través de la mediación y la resolución de conflictos
}

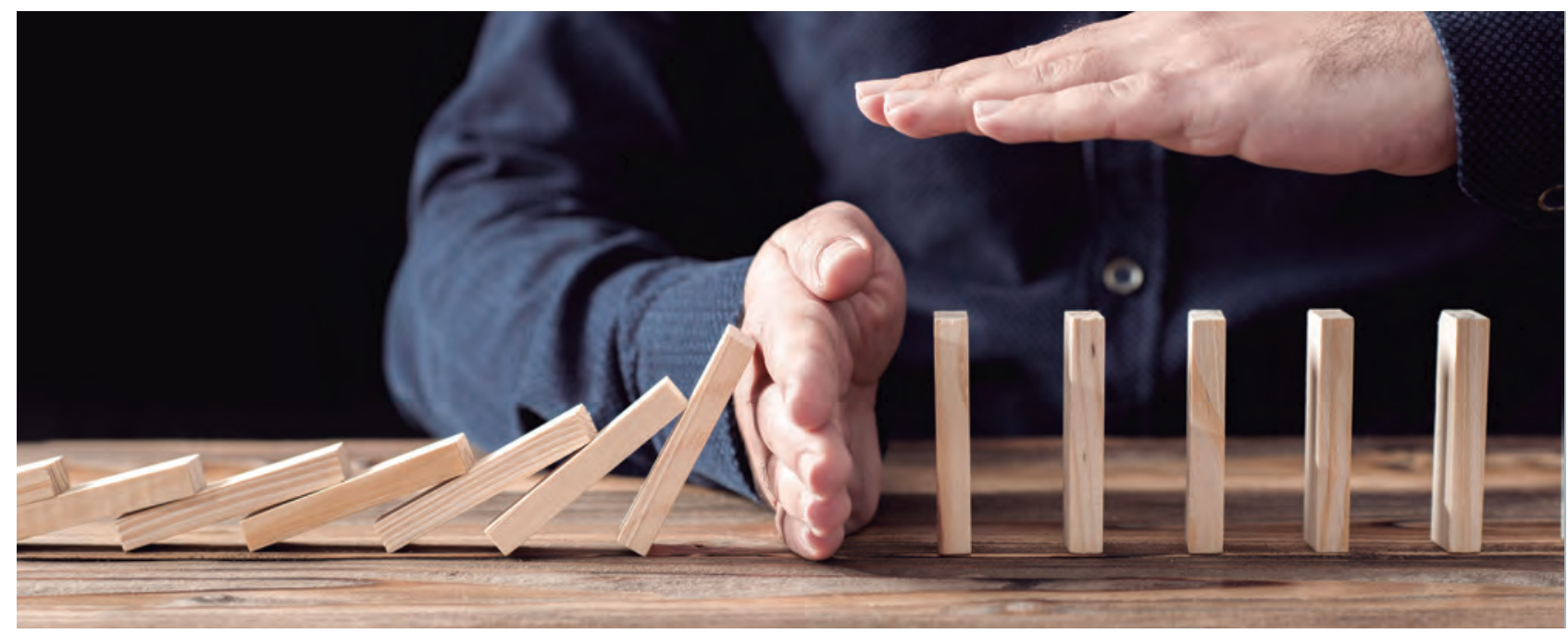

La mediación escolar conlleva un aprendizaje activo y experiencial que capacita para la convivencia positiva al mismo tiempo que permite el desarrollo de alumnos y profesores. El objetivo de este estudio es conocer cuál es el impacto de la mediación que las personas implicadas han percibido personalmente y en su entorno de convivencia. Los resultados ponen de relieve la percepción de aprendizajes emocionales, sociocognitivos y morales y la mejora de la

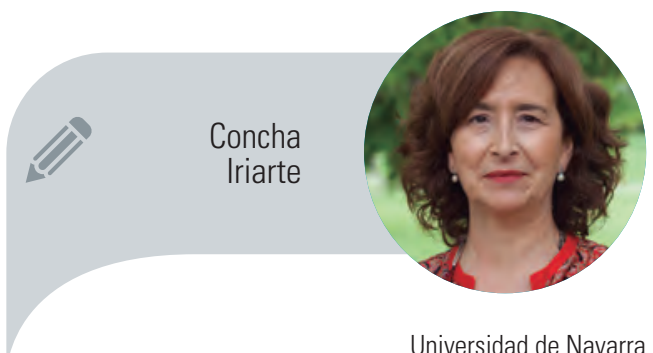
ciriarte@unav.es

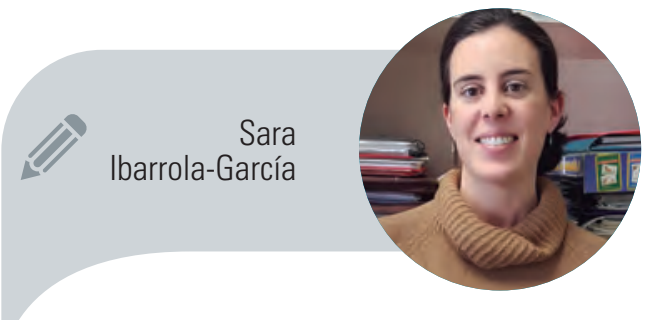

Universidad de Navarra sigarcia@unav.es convivencia en el centro educativo. 


\section{Introducción}

El ritmo frenético, la inestabilidad, el aluvión informativo y la superficialidad de las relaciones interpersonales desarrolladas por los adultos conforman el patrimonio que transferimos a las nuevas generaciones. De ahí que los centros (educativos) se hayan convertido en un hervidero de conflictos (CARRAsco, HerNÁNDEZ Y RIPOLL, 2012).

Es una apreciación dura, pero no exenta de cierta verdad. A su vez, la manera en que los niños afrontan los conflictos, en el día a día, es clave para su desarrollo emocional, sociocognitivo y moral presente y futuro. Si no se les orienta en la solución de los conflictos de manera constructiva, pueden desarrollar estrategias de resolución inefectivas y destructivas, tales como el empleo de la fuerza física, la agresividad verbal, la venganza o la evitación (TURNUKLU, KACMAZ, TURK y COLS., 2009). Nix y Hale (2007) asocian la impericia para manejar conflictos con el aumento del riesgo de abuso de sustancias, conductas violentas y baja autoestima entre los jóvenes. De hecho, la delincuencia y la violencia se han considerado síntomas de la falta de habilidad para afrontar constructivamente los conflictos en la edad escolar y juvenil (Jorbozeh, Dehdari, AshoORkHANI Y TAghdisI, 2014; Kapusuzoglu, 2010). Si lo vemos desde un punto de vista más positivo, sabemos que niños, adolescentes y adultos jóvenes que se entrenan en la resolución constructiva de conflictos, tienden a hacer y mantener más amigos, son más empleables, tienen más éxito en sus carreras, una vida familiar más satisfactoria, y generalmente experimentan menos estrés y más felicidad. De hecho, los estudios indican que enseñar a resolver los conflictos reduce significativamente la agresividad, tanto reactiva como proactiva en lo que son comportamientos frecuentes como hacer burla, gritar al otro, luchar, golpear o tirar del pelo (COOK y BOEs, 2013).

En este contexto, se ha demostrado que la mediación es una estrategia eficaz para el tratamiento de los conflictos, así lo consideran el $71 \%$ de los centros acogi-

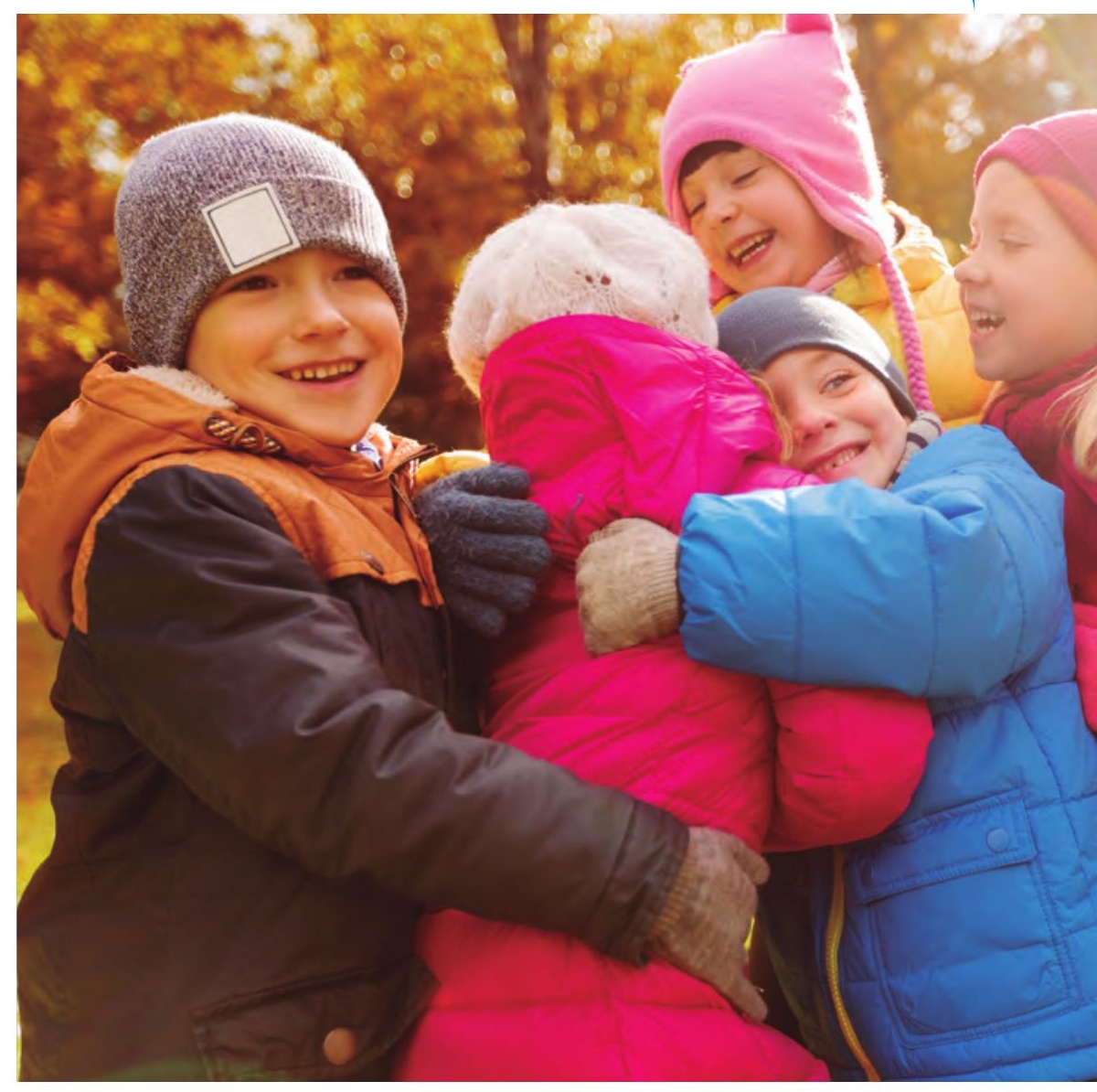

dos al proyecto de la investigación de Tórrego y Galán en 2008. Cook y Boes (2013) comprueban, a su vez, que la mediación entre iguales sirve para que los estudiantes tomen un papel activo en la creación de un mejor clima académico, disminuyendo el número de peleas y el absentismo escolar. Asimismo, en la evaluación del impacto de la mediación escolar que hacen Jorbozeh, Dehdari, Ashoorkhani y Taghdisi (2014), se observa la mejora de la empatía y la moral en el grupo y de la comprensión de las diferencias interpersonales, lo que genera una atmósfera más segura, alegre, de apoyo y confianza con los iguales en el centro educativo. Por todo lo cual, la mediación es una posibilidad efectiva de resolución de conflictos, y además promotora de valores cívicos propios de una ciudadanía responsable.

La mediación capacita cívicamente a los alumnos, los prepara para ser ciudadanos activos en el futuro, pero, especialmente, les facilita el compromiso democrático en el presente (SelLman, 2011). En este sentido, es una oportunidad para empoderar a los alumnos al transferirles poder y autoridad. Nix y Hale (2007) 
Modelo educativo de desarrollo de competencias a través de la mediación (MEDECOME) Ibarrola-García y Iriarte (2012)

\begin{tabular}{|c|c|c|}
\hline $\begin{array}{l}\text { COMPETENCIA } \\
\text { EMOCIONAL }\end{array}$ & $\begin{array}{l}\text { COMPETENCIA } \\
\text { SOCIOCOGNITIVA }\end{array}$ & $\begin{array}{l}\text { COMPETENCIA } \\
\text { MORAL }\end{array}$ \\
\hline $\begin{array}{l}\text { yAsertividad } \\
\text { У Autoconciencia } \\
\text { emocional } \\
\text { y Autorregulación } \\
\text { emocional } \\
\text { y Empatía }\end{array}$ & $\begin{array}{l}\text { צPensamiento } \\
\text { de solución } \\
\text { de problemas } \\
\text { interpersonales } \\
\text { У Conocimiento } \\
\text { acerca de } \\
\text { la teoría de } \\
\text { resolución de } \\
\text { conflictos } \\
\text { У Técnicas } \\
\text { comunicativas }\end{array}$ & $\begin{array}{l}\text { У Respeto hacia } \\
\text { los otros y } \\
\text { reciprocidad } \\
\text { У Atribución de } \\
\text { responsabilidad } \\
\text { У Razonamiento } \\
\text { moral y toma de } \\
\text { decisiones }\end{array}$ \\
\hline
\end{tabular}

han visto, incluso, que la mediación entre iguales, con plena autonomía, da a los profesores más tiempo para concentrarse en sus tareas académicas y a los alumnos mayor implicación y protagonismo en la salvaguarda de la convivencia. Asimismo, la mediación aumenta los comportamientos constructivos en el colegio, en casa y en la comunidad, desarrolla valores prosociales (Jones, 2004), reduce las sanciones, el absentismo, y aumenta la confianza en uno mismo frente a la tarea, así como el rendimiento académico (KAPUsuzogLu, 2010); también mejora la empatía (TURnuklu, Kacmaz, Gurler, Kalender, Zengin y SEVKIN, 2009). En suma, favorece la madurez psicológica (Jorbozeh, Dehdari, AsHOORKHANI Y TAGHDISI, 2014), convirtiéndose en una opción valiosa y un recurso potente para mejorar la calidad educativa.

\section{Evidencias en nuestra investigación}

Desde el año 2011 las autoras venimos investigando sobre el impacto positivo que produce la mediación tanto en los profesores y alumnos mediadores como en los alumnos mediados, así como la influencia en el contexto social del centro y de la vida de los participantes en mediación.

A partir del modelo MEDECOME sobre capacitación emocional, sociocognitiva y moral que aparece en la tabla 1, se di- señaron y validaron tres cuestionarios, publicados en el libro La convivencia escolar en positivo. Mediación y Resolución de Conflictos (Pirámide, 2012), que nos han permitido, a lo largo de estos años, conocer mejor la influencia capacitadora de la mediación. Pueden consultarse estas investigaciones con más detalle en Ibarrola-García y Iriarte (2012, 2013 a,b,c; 2014 a,b,c); Ibarrola-García, Iriarte y Aznárez-Sanado (2017); Iriarte y IbarrolaGarcía (2017). Se muestra a continuación una síntesis de los principales resultados obtenidos.

\section{Aprendizaje sociocognitivo percibido por los alumnos mediadores y mediados y por los profesores mediadores tras participar en mediación}

Los alumnos mediadores perciben, como cambio principal y significativo, la objetividad al analizar y resolver los conflictos, es decir, la mediación les ha ayudado a analizar los conflictos sin dejarse llevar por su opinión personal. En segundo lugar, destacan el pensamiento alternativo, esto es, han aprendido a pensar más en alternativas diferentes para solucionar los problemas.

En los alumnos mediados, por su parte, destacan como aprendizajes sociocognitivos principales la ayuda de la mediación para mejorar la comunicación con los demás, también perciben un aumento del pensamiento consecuencial al analizar los conflictos (han aprendido a anticipar cognitivamente las consecuencias de sus acciones), y el pensamiento medios-fines (han aprendido a pensar si con su conducta conflictiva consiguen realmente lo que pretenden).

En el profesor mediador destaca especialmente la capacidad de la mediación para fomentar el pensamiento alternativo, es decir, los profesores valoran la ayuda que ha supuesto la mediación para pararse a pensar en diferentes formas de solución ante los conflictos. En segundo lugar, han mejorado su capacidad de analizar los conflictos con objetividad sin dejarse llevar por su opinión. 
Contenidos de los ítems de la dimensión "Percepción de aprendizaje socioafectivo" en los cuestionarios del alumno mediador y del alumno mediado (Ibarrola-García y lriarte, 2012)

\section{Aspectos emocionales}

La mediación me ha ayudado a comprender mejor el punto de vista de los demás

La mediación me ha ayudado a expresar mejor mis necesidades, sentimientos y emociones en la relación con los demás

La mediación me ha ayudado a darme cuenta de cuáles son mis emociones negativas

La mediación me ha ayudado a controlar mejor las emociones negativas (sus causas, consecuencias, intensidad o duración)

La mediación me ha hecho darme cuenta de que mis emociones influyen en cómo me comporto

La mediación me ha ayudado a darme cuenta de que a veces hay diferencias entre lo que siento, lo que pienso y mis valores

La mediación me ha ayudado a reconocer emociones en los gestos, movimientos o expresiones de los demás

La mediación ha aumentado mi paciencia ante los problemas

La mediación me ha ayudado a señalar a otros si han actuado de forma injusta

La mediación me ha ayudado a comunicarme mejor con los demás

** En el cuestionario del alumno mediador también se incluye: Como mediador me resulta difícil identificar qué emociones tienen las personas en conflicto

\section{Aspectos sociocognitivos}

Antes veía el conflicto como algo negativo y ahora lo veo como una oportunidad para aprender

He aprendido a analizar los conflictos sin dejarme llevar por mi opinión personal (comprendiendo sus causas, lo que sienten y piensan las personas...)

Me paro a pensar más en las consecuencias que puede tener no actuar bien

En un conflicto me paro a pensar más en diferentes formas de solucionarlo

Me paro a pensar más si con mi conducta he conseguido realmente lo que pretendía

**En el cuestionario del alumno mediador también se incluye: Utilizo con más frecuencia técnicas comunicativas en mi vida diaria (ejemplo: formulación de preguntas, parafraseo, mensajes en primera persona, etc.)

\section{Aspectos morales}

La mediación me ha hecho darme cuenta de la responsabilidad que tengo en la solución de mis propios conflictos Soy más respetuoso y acepto mejor las diferencias de las personas con las que me relaciono

Me siento más implicado en el día a día de mi colegio y especialmente en situaciones en las que puedo participar Considero que la mediación me ha ayudado a comprender mejor lo que es justo y lo que es injusto Desde que soy mediador doy más importancia a no hablar mal de las personas cuando no están Ahora doy más importancia a lo que puede hacer cada uno para que haya buena convivencia

**En el cuestionario del alumno mediador también se incluye: Ahora valoro más la importancia de saber pedir ayuda cuando se necesita

\section{Aprendizaje moral percibido por los alumnos mediadores y mediados y por los profesores mediadores}

En cuanto a los aprendizajes morales, los alumnos mediadores han descubierto con la mediación: la importancia de las relaciones de ayuda, especialmente, la importancia de saber pedir ayuda antes de que sea tarde. En segundo lugar, la acep- tación y el respeto de las diferencias de las personas con las que uno se relaciona $y$, en tercer lugar, la influencia que cada persona ejerce en el conjunto del clima social, descubriendo la aportación significativa de cada uno en pro de la mejora de la convivencia.

Por su parte, los alumnos mediados valoran sobre todo la mejora de la confiabilidad, es decir, después de participar 


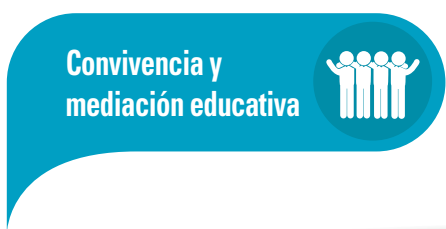

Investigaciones actuales sobre los beneficios que promueve la mediación

ע Impulsa la convivencia (Carrasco y Cols, 2012)

\ Intensifica la adquisición de competencias (Carrasco y Cols., 2012)

$\searrow$ Los alumnos toman un rol participante-activo (Cook y Boes, 2013)

$\checkmark$ Disminuye el conflicto y el absentismo (Cook y BoEs, 2013)

$\searrow$ DeVoogd, Lane-Garon y Kralowec (2016)

$\checkmark$ Los alumnos experimentan un crecimiento social y emocional superior

$\checkmark$ Los alumnos mejoran la perspectiva cognitivo-emocional, el rendimiento académico, la sensación de seguridad y pertenencia, eligen estrategias de solución de conflictos más adecuadas

y Aumenta la empatía

У Jorbozeh, Dehdari, Ashoorkhan y Tagholsi (2004), aumenta:

$\searrow$ La moral en el grupo

$\searrow$ La comprensión de las diferencias interpersonales

У El número de amigos

$\searrow$ La autoestima

$\searrow$ La participación

$\searrow$ La responsabilidad

$\checkmark$ El aprendizaje

\section{Aprendizajes emocionales percibidos por los alumnos mediados y mediadores y los profesores mediadores}

Tanto los profesores y alumnos mediadores, como los alumnos mediados, perciben importantes cambios emocionales, lo que parece indicar la efectividad del proceso de mediación en este sentido. Todos ellos notan una mejora en su conciencia y regulación de las emociones, aunque de forma más notable en esta última. En resumen, podemos decir que la mediación ha ayudado a comprender mejor el punto de vista de los demás, a detectar las emociones negativas, conocer la influencia del impacto de las emociones en el comportamiento, lo que facilita la comprensión, expresión y comunicación emocional.

\section{Mejoras percibidas en la convivencia en el centro educativo}

La mediación ha abierto cauces en los en mediación dan más importancia a no hablar mal de otras personas cuando no están presentes. También la mejora del sentido de justicia, comprendiendo mejor lo que es justo y lo que es injusto; y, en tercer lugar, la disposición a pedir ayuda para evitar o resolver problemas. Asimismo, valoran especialmente la importancia de la mediación para aminorar la escalada de los conflictos en el centro educativo, para ofrecer apoyo social a personas en conflicto o en riesgo de conflicto, y para aumentar la aceptación y el respeto de las diferencias entre todos.

Con relación al profesor, los tres aspectos más valorados están relacionados con la contribución de este a su entorno en la mejora de la convivencia a raíz de haber participado en procesos de mediación. Los profesores mediadores perciben un incremento de su participación en el colegio, dan más importancia a lo que puede hacer cada uno para que haya una buena convivencia y a la responsabilidad que tienen en la solución de los conflictos. La mediación incide positivamente sobre la tarea cívica del profesor aportando un motivo para ejercitarla. centros educativos para la participación de alumnos y profesores en la mejora de la convivencia, ha mejorado la cultura de la colaboración, constatando que es un apoyo social relevante tanto para alumnos como para profesores. Asimismo, la mediación ha permitido aumentar el respeto hacia las diferencias entre los compañeros, lo que sin duda facilita la inclusión.

\section{Conclusiones}

1. La mediación educativa es una estrategia, no solamente eficaz para transformar conflictos, sino que encierra un importante potencial formativo tanto individual como interpersonal de los agentes implicados en ella, así como en el conjunto del clima de convivencia de los centros educativos.

2. En nuestra investigación, relativa a la evaluación del proceso de mediación en población española, se corroboran estas afirmaciones en el sentido de que:

$\boldsymbol{\gamma}$ Los alumnos han descubierto la utilidad de la mediación y su importancia en el desarrollo de relaciones, fomentando la aceptación 


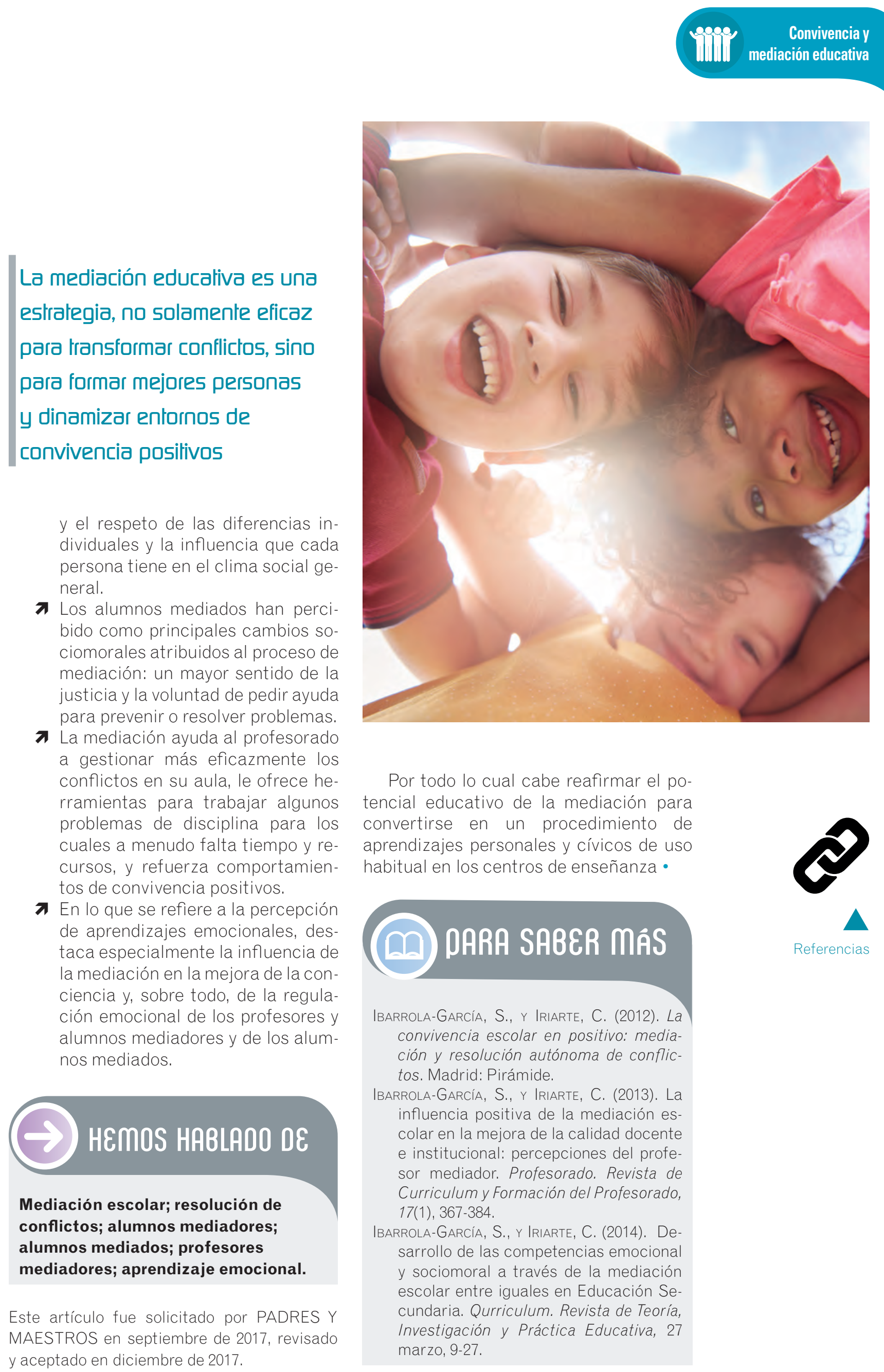

